\title{
The prognostic value of lymph node ratio and log odds of positive lymph nodes in patients with lung adenocarcinoma
}

\author{
Yue Zhao, MD, ${ }^{\mathrm{a}, \mathrm{b}}$ Guodong Li, MD, ${ }^{\mathrm{b}, \mathrm{c}}$ Difan Zheng, MD, ${ }^{\mathrm{a}, \mathrm{b}}$ Ming Jia, MD, ${ }^{\mathrm{a}, \mathrm{b}}$ Weixing Dai, MD, ${ }^{\mathrm{b}, \mathrm{d}}$ \\ Yihua Sun, MD, ${ }^{\mathrm{a}, \mathrm{b}}$ and Haiquan Chen, MD ${ }^{\mathrm{a}, \mathrm{b}}$
}

\section{ABSTRACT}

Objective: To investigate whether lymph node ratio and log odds ratio can be used for predicting the prognosis of patients with lung adenocarcinoma.

Methods: A total of 1097 patients with lung adenocarcinoma who underwent complete surgical resection and systematic lymph node dissection between 2008 and 2013 were studied retrospectively. Lymph node ratio was calculated as: $\frac{\text { positive lymph nodes }}{\text { total lymph nodes }}$. Log odds ratio was calculated as:

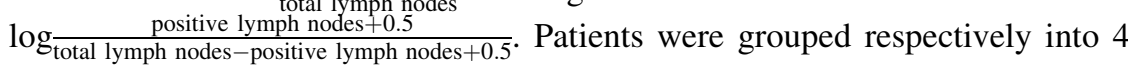
groups by the use of recursive partitioning according to their lymph node ratio and $\log$ odds ratio. Linear trend $\chi^{2}$ test was used for measuring monotonicity and discriminatory power.

Results: The 3-year survival rate was $91.0 \%(89.9 \%-92.1 \%)$ for group $0,75.2 \%$ $(71.4 \%-79.0 \%)$ for group $1,56.5 \%(51.5 \%-61.5 \%)$ for group 2 , and $41.4 \%$ $(36.4 \%-46.4 \%)$ for group 3 of lymph node ratio, respectively. In addition, the 3-year survival rate was $89.9 \%(88.7 \%-91.1 \%)$ for group $1,78.4 \%$ (74.4\%-82.4\%) for group 2, 56.0\% (50.9\%-61.1\%) for group 3, and $41.0 \%$ $(36.1 \%-45.9 \%)$ for group 4 of $\log$ odds ratio, respectively. Univariable and multivariable Cox models identified that both lymph node ratio and log odds ratio were significant prognostic factors for patients' survival. Log-rank test of trend $\chi^{2}$ statistics of both lymph node ratio $(P<.001)$ and log odds ratio $(P<.001)$ showed significant differences.

Conclusions: Both lymph node ratio and log odds ratio can be used as prognostic factors for clinicians to predict patients' prognosis. (J Thorac Cardiovasc Surg 2017;153:702-9)

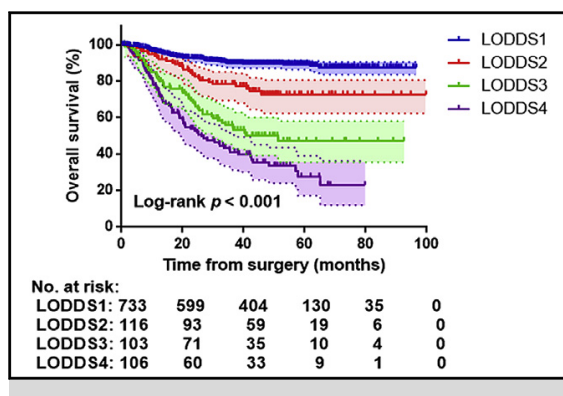

Overall survival grouped by the log odds ratio system.

\section{Central Message}

Both lymph node ratio and log odds ratio of positive lymph nodes are of prognostic value for patients' prognosis. They are worth considering when making therapeutic strategies.

\section{Perspective}

Our study reveals that although the number of metastatic lymph nodes plays no role in determining patients' $\mathrm{N}$ stage in lung cancer, it still has the prognostic value. Both lymph node ratio and log odds ratio of positive lymph nodes are good prognostic indices for lung cancer.

See Editorial Commentary page 710.
The prognosis for patients with non-small cell lung cancer currently is estimated based on the tumor, node, metastasis (TNM) system, which assigns nodal metastasis based on the involved lymph node station. The determination of the $\mathrm{N}$ stage of non-small cell lung cancer was based

From the Departments of ${ }^{\mathrm{a}}$ Thoracic Surgery, ${ }^{\mathrm{c}}$ Interventional Therapy, and ${ }^{\mathrm{d}}$ Colorectal Surgery, Fudan University Shanghai Cancer Center; and ${ }^{\mathrm{b}}$ Department of Oncology, Shanghai Medical College, Fudan University, Shanghai, China.

Funded by the National Natural Science Foundation of China (81330056, 81401886, $81401891,81422029,81472173$, and 81372525) and the Key Project of Science and Technology Commission of Shanghai Municipality (JGGG1302).

Drs Zhao and Li contributed equally to this work.

Received for publication May 30, 2016; revisions received Nov 6, 2016; accepted for publication Nov 24, 2016.

Address for reprints: Haiquan Chen, MD, Department of Thoracic Surgery, Fudan University Shanghai Cancer Center, 270 Dong-An Rd, Shanghai 200032, China (E-mail: hqchen1@yahoo.com); and Yihua Sun, MD, Department of Thoracic Surgery, Fudan University Shanghai Cancer Center, 270 Dong-An Rd, Shanghai 200032, China (E-mail: Sun_yihua76@ hotmail.com).

$0022-5223 / \$ 36.00$

Copyright (c) 2016 by The American Association for Thoracic Surgery

http://dx.doi.org/10.1016/j.jtcvs.2016.11.053 mainly on the lymphatic region involved, according to the seventh and the upcoming eighth TNM staging system. ${ }^{1,2}$ This current approach does not take into consideration the number of involved lymph nodes, however, which may be limiting the precision of the prognostic estimates.

Although the number of metastatic lymph nodes plays no role in determining the $\mathrm{N}$ stage of a primary lung cancer, several studies have found that patients with a high lymph node ratio (LNR) tend to have a poor prognosis compared with those with a low LNR. ${ }^{3-9}$ Moreover, log odds ratio (LODDS), defined as the $\log$ of the ratio between the number of positive lymph nodes (PLNs) and the number of negative lymph nodes, has been proved effective in

Scanning this QR code will take you to supplemental tables and video for this article. 


$$
\begin{aligned}
& \text { Abbreviations and Acronyms } \\
& \begin{array}{ll}
\text { AIC } & =\text { Akaike information criterion } \\
\text { IQR } & =\text { interquartile range } \\
\text { LNR } & =\text { lymph node ratio } \\
\text { LODDS } & =\text { log odds ratio } \\
\text { LR } & =\text { likelihood ratio } \\
\text { OS } & =\text { overall survival } \\
\text { PLN } & =\text { positive lymph node } \\
\text { pN } & =\text { pathologic N stage } \\
\text { RFS } & =\text { recurrence-free survival } \\
\text { TNM } & =\text { tumor, node, metastasis }
\end{array}
\end{aligned}
$$

predicting the prognosis of patients with head and neck, breast, gallbladder, gastric, and colorectal cancers. ${ }^{10-14}$ Whether LODDS can be used as a prognostic index of non-small cell lung cancer, however, is still unclear.

Our study aims to determine the discriminatory accuracy of LODDS for prognosis after surgical resection in patients with resectable non-small cell lung cancer. Our secondary aim was to compare the prognostic stratification of LNR and LODDS with the current $\mathrm{N}$ staging system.

\section{PATIENTS AND METHODS Patients}

A total of 1097 patients with clinically resectable lung adenocarcinoma who did not have invasive mediastinal staging between May 2008 and May 2013 in Fudan University Shanghai Cancer Center were included in this study (Figure 1). All patients received a surgical R0 resection and systematic lymph node dissection. The modality of skeletal systematic lymph node dissection was performed according to the tumor location. Lymph node stations $7,8,10$, and 11 were dissected routinely regardless of the tumor location. In addition, for a tumor located in the left upper lobe, lymph node stations 4, 5, and 6 were dissected routinely. For a tumor located in the left lower lobe, lymph node stations 4, 5, 6, and 9 were dissected routinely. For a tumor located in the right upper lobe, lymph node stations 2 and 4 were dissected routinely, and station 3 was dissected optionally. For a tumor located in the right middle lobe, lymph node stations 2 and 4 were dissected routinely, and station 3 was dissected optionally. For a tumor located in the right lower lobe, lymph node stations 2, 4, and 9 were dissected routinely, and station 3 was dissected optionally (Video 1). Patients with stage II or III diseases received a 4-cycle, platinum-based doublet chemotherapy 3 to 4 weeks after surgery. No patients underwent neoadjuvant therapy. None of the included patients received invasive mediastinal staging before surgery. Resected tumors and lymph nodes were labeled in the operation room and reviewed by pathologists to determine their pathologic stage, according to the current seventh TNM staging system for lung cancer. ${ }^{1}$ All lymph nodes resected were identified and reported by pathologists. This study was approved by the Committee for Ethical Review of Research (Fudan University Shanghai Cancer Center IRB\# 090977-1). Patients' informed consents were waved because this was a retrospective study.

\section{Follow-up Protocol}

The start point of a patient's follow-up data is considered the day he/she received surgery. Patients came to hospital to take a routine

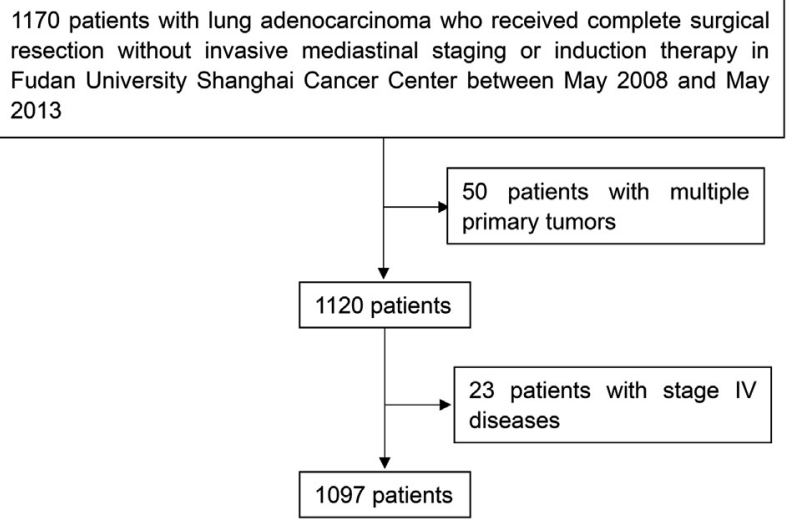

FIGURE 1. Inclusion and exclusion criteria.

examination including chest computed tomography, brain magnetic resonance imaging, and abdominal ultrasonography every 3 months for the first 2 years after surgery, every 6 months for the next 2 years after surgery, and once a year from then on. Recurrence was defined as local if it was at the site of the excised lobe (including infiltrations along the stapler line and stumps of bronchi and vessels), regional if it involved mediastinal or hilar lymph nodes, or an ipsilateral lobe, distal if it occurred at other organs or as a pleural diffuse. A telephone follow-up would be made if the patient didn't come to the clinic. The follow-up work started in May 2008 and ended in October 2016. Overall survival (OS) was considered as the time between the day of surgery and the day of death from any cause. Recurrence-free survival (RFS) was considered as the time between the day of surgery and the day of disease recurrence. Patients who died before disease recurrence were censored on the date of their last day alive and patients with no events were censored at the date of their last follow-up.

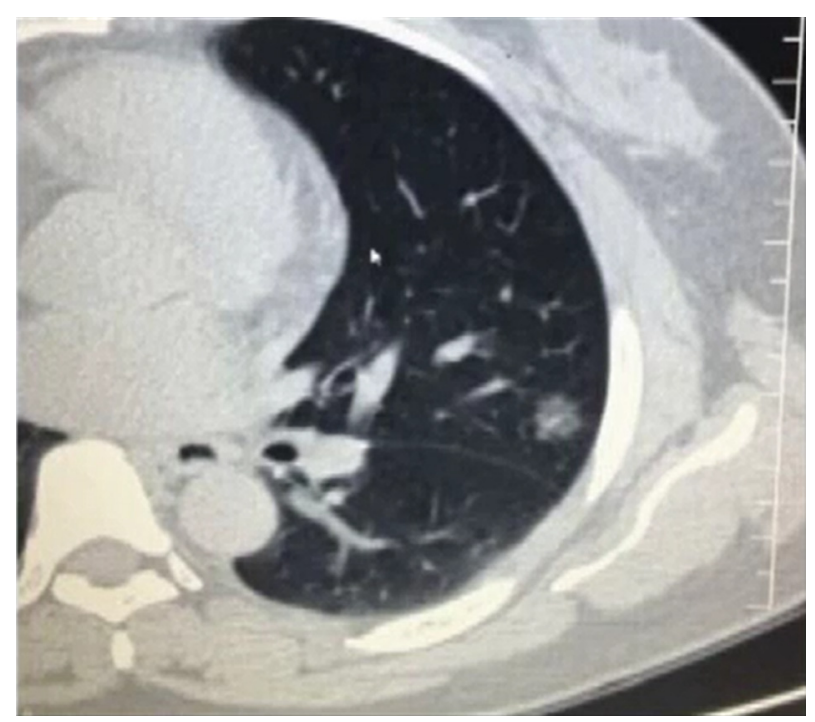

VIDEO 1. Left upper lobe-lingual segmentectomy plus systematic lymph node dissection. Video available at: http://www.jtcvsonline.org/article/ S0022-5223(16)31672-5/addons. 


\section{Definition of LNR and LODDS}

LNR was defined as the ratio between the number of PLNs and the number of total lymph nodes retrieved. LNR was divided into 4 groups by the use of recursive partitioning: LNR0 (LNR = 0), LNR1 $(0<$ LNR $\leq 0.14)$, LNR2 $(0.14<$ LNR $\leq 0.38)$, and LNR3 $(0.38<$ LNR $\leq 1)$. LODDS was defined as the log ratio between the number of PLNs and the number of negative lymph nodes, it was calculated as: $\log \frac{\mathrm{PLN}+0.5}{\mathrm{TLN}-\mathrm{PLN}+0.5}$, where 0.5 was added into both the numerator and the denominator to avoid singularity. ${ }^{15}$ Based on the LODDS calculated, LODDS was divided into 4 groups using recursive partitioning: LODDS1 $(-2.10 \leq$ LODDS $\leq-1.01)$, LODDS2 $(-1.01<$ LODDS $\leq-0.70)$, LODDS3 $(-0.70<$ LODDS $\leq-0.20)$, and LODDS4 $(-0.20<$ LODDS $\leq 1.74)$.

\section{Statistical Analyses}

Clinical and pathologic characteristics, including patient sex, age, smoking status, pathologic $\mathrm{T}$ stage, pathologic $\mathrm{N}$ stage, pathologic TNM stage, lymphovascular invasive status, number of lymph nodes resected, number of positive nodes, Charlson comorbidity index, surgery type, tumor location, LNR, and LODDS were recorded. Log-rank test was performed to analyze the correlation between patients' clinicopathologic characteristics and 3-year OS. Number of lymph nodes resected was dichotomized with the receiver operating characteristic curves, where 14.50 was chosen as the cut-point where the predictive performance was the highest. Number of positive nodes was grouped by the use of recursive partitioning. Kaplan-Meier survival curves were used to compare the RFS and OS between different groups of patients. Log-rank test of trend $\chi^{2}$ statistics were used to assess discriminatory ability and monotonicity. In addition, we performed a 2-step univariable and multivariable Cox proportional hazards regression model to identify independent prognostic factors for patients with lung adenocarcinoma. First, univariable analysis was performed to identify which among those confounding factors, including sex, age, smoking status, surgery type, Charlson comorbidity index, percent of forced expiratory volume in 1 second predicted, adjuvant chemotherapy, pathologic $\mathrm{T}$ stage, pathologic $\mathrm{N}$ stage, TNM stage, tumor location, number of lymph nodes resected, number of PLNs, LNR, and LODDS, were prognostic factors. Second, we separately included $\mathrm{N}, \mathrm{LNR}$, and LODDS in 3 multivariable Cox regression models, along with those factors with a $P$ value $<.1$ in the first step to identify independent prognostic factor(s). The Spearman rank correlation was used to assess the correlation between LNR and LODDS. Model fit was examined with the Akaike Information Criterion (AIC), and the Harrell C-statistic was used to assess discriminatory accuracy of each model. All statistical analyses were performed with SPSS (version 22.0; IBM Corp, Armonk, NY) and R (version 3.3.0; R Foundation for Statistical Computing, Vienna, Austria). Survival curves were depicted using GraphPad Prism (version 6.01; GraphPad Software Inc, La Jolla, Calif).

\section{RESULTS}

For all the 1097 patients, the median number of lymph node stations dissected was 6 , and the median number of lymph nodes dissected was 16 . The median follow-up time was 40.0 (interquartile range [IQR], 22.2-51.6) months. Of them, 729 were in the LNR0 group, 157 were in the LNR1 group, 101 were in the LNR2 group, and 110 were in the LNR3 group. For the LODDS system, 762 were in the LODDS1 group, 118 were in the LODDS2 group, 106 were in the LODDS3 group, and 111 were in the LODDS4 group (Table 1). The median follow-up time was 41.5 (IQR, 27.4-53.3), 40.8 (IQR, 23.1-52.8), 32.0 (IQR,
13.4-45.0), and 23.4 (IQR, 11.7-42.3) months for patients in the LNR0, LNR1, LNR2, and LNR3 groups. The median follow-up time was 41.5 (IQR, 27.3-54.2), 40.0 (IQR, 23.2-50.1), 31.6 (IQR, 13.2-44.9), and 23.2 (IQR, 11.8-42.2) months for patients in the LODDS1, LODDS2, LODDS3, and LODDS4 groups. The relationship between clinicopathologic characteristics and 3-year OS was shown in Table 1. Patients who were male, former/current smokers, with lymphovascular invasion, with more lymph nodes dissected, with more positive nodes examined, with a greater Charlson comorbidity index, who received more invasive surgery, and received adjuvant chemotherapy tended to have a lower 3-year survival rate. In addition, $\mathrm{T}$ stage, $\mathrm{N}$ stage, TNM stage, LNR group, and LODDS group had a significant influence on patients' 3-year OS. Basically, the greater the stage, the poorer the prognosis. Each variable in Table 1 also was stratified by the LODDS strata, and the distribution of pathologic $\mathrm{N}$ stage $(\mathrm{pN})$ in the LNR strata is shown in Table E1.

RFS and OS for pN, LNR, and LODDS were analyzed by the use of Kaplan-Meier survival curves, which are shown in Figure 2 and Figure 3. All 3 systems were associated strongly with patients' RFS (Figure 2) and OS (Figure 3). Log-rank test of trend $\chi^{2}$ statistics showed $\mathrm{pN}(P<.001)$, LNR $(P<.001)$, and LODDS $(P<.001)$ showed good discriminatory ability and monotonicity. Among 426 patients with recurrent diseases, local recurrence was seen in 22 patients, regional recurrence was seen in 90 patients, distal recurrence was seen in 221 patients, and data of recurrent site for 93 patients were unavailable. A comparison between single or multiple lymph node stations involved in patients with $\mathrm{N} 1$ and $\mathrm{N} 2$ diseases is shown in Table E2. As shown in Figures 2 and 3, there were significant differences in patients' RFS and OS in all 3 systems. Moreover, we used Cox proportional hazards regression model to assess whether this result could be influenced by other clinical and pathologic factors. In the univariable Cox model, the results showed that sex, smoking status, surgery type, percent of forced expiratory volume in 1 second predicted, adjuvant chemotherapy, pathologic $\mathrm{T}$ stage, pathologic $\mathrm{N}$ stage, pathologic TNM stage, tumor location, total number of lymph nodes resected, number of PLNs, and LNR and LODDS were all prognostic factors (Table 2). Then, in the multivariable analysis 1, when put all the clinical and pathologic risk factors together, both $\operatorname{LNR}(P=.073)$ and LODDS $(P=.046)$ were shown to be independent prognostic factors, whereas $\mathrm{pN}$ stage failed to show a significant difference $(P=.284$, Table 2$)$.

With the previous results strongly supporting the point that LNR and LODDS were able to predict patients' prognoses, we next performed likelihood ratio (LR) test, calculated the Harrell Concordance index and AIC of pN, LNR, and LODDS systems. The results are shown in 
TABLE 1. Clinicopathologic characteristics of patients with lung adenocarcinoma $(n=1097)$

\begin{tabular}{|c|c|c|c|c|c|c|c|}
\hline & $\begin{array}{c}\text { No. } \\
\text { cases }\end{array}$ & $\begin{array}{c}\text { LODDS1 } \\
(-\mathbf{2 . 1 0}<\text { LODDS } \\
\leq-\mathbf{1 . 0 1})\end{array}$ & $\begin{array}{c}\text { LODDS2 } \\
(-\mathbf{1 . 0 1}<\text { LODDS } \\
\leq-\mathbf{0 . 7 0})\end{array}$ & $\begin{array}{c}\text { LODDS3 } \\
(-\mathbf{0 . 7 0}<\text { LODDS } \\
\leq-\mathbf{0 . 2 0})\end{array}$ & $\begin{array}{c}\text { LODDS4 } \\
(-0.20<\text { LODDS } \\
\leq 1.74)\end{array}$ & $\begin{array}{l}3-y \text { OS } \% \\
(95 \% \text { CI) }\end{array}$ & $\begin{array}{c}P \text { value } \\
\text { (log-rank } \\
\text { test) }\end{array}$ \\
\hline \multicolumn{8}{|l|}{ Sex } \\
\hline Male & 609 & 439 & 67 & 51 & 52 & $76.3(74.2-78.4)$ & .004 \\
\hline Female & 488 & 323 & 51 & 55 & 59 & $83.1(81.5-84.7)$ & \\
\hline \multicolumn{8}{|l|}{ Age group, y } \\
\hline$\leq 60($ mean age $)$ & 541 & 355 & 61 & 59 & 66 & $81.7(79.9-83.5)$ & .326 \\
\hline$>60$ & 556 & 407 & 57 & 47 & 45 & $78.5(76.6-80.4)$ & \\
\hline \multicolumn{8}{|l|}{ Smoking status } \\
\hline Former/current & 353 & 227 & 37 & 50 & 39 & $71.6(69.0-74.2)$ & $<.001$ \\
\hline Never & 744 & 535 & 81 & 56 & 72 & $84.1(82.7-85.5)$ & \\
\hline \multicolumn{8}{|l|}{$\mathrm{T}$ stage } \\
\hline 1 & 638 & 493 & 60 & 46 & 39 & $89.1(87.8-90.4)$ & $<.001$ \\
\hline 2 & 384 & 231 & 55 & 47 & 51 & $72.7(70.3-75.1)$ & \\
\hline 3 & 56 & 31 & 3 & 9 & 13 & $42.3(35.1-49.5)$ & \\
\hline 4 & 19 & 7 & 0 & 4 & 8 & $49.9(38.0-69.8)$ & \\
\hline \multicolumn{8}{|l|}{$\mathrm{N}$ stage } \\
\hline 0 & 729 & 702 & 22 & 5 & 0 & $91.0(89.9-92.1)$ & $<.001$ \\
\hline 1 & 108 & 30 & 49 & 17 & 12 & $83.5(79.7-87.3)$ & \\
\hline 2 & 260 & 30 & 47 & 84 & 99 & $50.8(47.6-54.0)$ & \\
\hline \multicolumn{8}{|l|}{ TNM stage } \\
\hline I & 663 & 635 & 23 & 5 & 0 & $94.1(93.1-95.1)$ & $<.001$ \\
\hline II & 142 & 80 & 37 & 15 & 10 & $75.5(41.5-79.5)$ & \\
\hline III & 292 & 47 & 58 & 86 & 101 & $53.1(50.1-56.1)$ & \\
\hline \multicolumn{8}{|l|}{ Lymphovascular invasion } \\
\hline Yes & 178 & 46 & 27 & 43 & 62 & $56.2(52.1-60.3)$ & $<.001$ \\
\hline No & 919 & 716 & 91 & 63 & 49 & $84.6(83.3-85.9)$ & \\
\hline \multicolumn{8}{|c|}{ Number of lymph nodes dissected } \\
\hline$<15$ & 443 & 314 & 48 & 39 & 42 & $85.8(84.0-87.6)$ & $<.001$ \\
\hline$\geq 15$ & 654 & 448 & 70 & 67 & 69 & $76.1(74.3-77.9)$ & \\
\hline Number of positive nodes & & & & & & & $<.001$ \\
\hline 0 & 729 & 702 & 22 & 5 & 0 & $91.0(89.9-92.1)$ & \\
\hline $1-2$ & 157 & 58 & 78 & 16 & 5 & $76.3(72.7-79.9)$ & \\
\hline $3-6$ & 101 & 2 & 18 & 58 & 23 & $54.8(49.6-60.0)$ & \\
\hline$>6$ & 110 & 0 & 0 & 27 & 63 & $39.5(34.5-44.5)$ & \\
\hline Charlson comorbidity index & & & & & & & $<.001$ \\
\hline 0 & 782 & 540 & 89 & 75 & 78 & $79.5(77.9-81.1)$ & \\
\hline 1 & 262 & 184 & 24 & 25 & 29 & $81.1(78.5-83.7)$ & \\
\hline 2 & 47 & 34 & 4 & 6 & 3 & $85.0(79.3-90.7)$ & \\
\hline 3 & 6 & 4 & 1 & 0 & 1 & $66.7(47.5-85.9)$ & \\
\hline Surgery type & & & & & & & $<.001$ \\
\hline Wedge/seg & 131 & 89 & 13 & 15 & 14 & $90.8(88.0-93.6)$ & \\
\hline Lob/bilobectomy & 946 & 667 & 102 & 86 & 91 & $79.7(78.3-81.1)$ & \\
\hline Pneumonectomy & 20 & 6 & 3 & 5 & 6 & $39.7(28.0-51.4)$ & \\
\hline Adjuvant chemotherapy & & & & & & & $<.001$ \\
\hline Yes & 445 & 186 & 84 & 83 & 92 & $68.4(66.0-70.8)$ & \\
\hline No & 652 & 576 & 34 & 23 & 19 & $88.3(87.0-89.6)$ & \\
\hline \multicolumn{8}{|l|}{ Tumor location } \\
\hline LUL & 291 & 186 & 38 & 30 & 37 & $78.6(76.0-81.2)$ & .483 \\
\hline LLL & 185 & 122 & 17 & 27 & 19 & $75.5(72.2-78.8)$ & .047 \\
\hline RUL & 352 & 263 & 37 & 28 & 24 & $84.2(82.1-86.3)$ & .094 \\
\hline RML & 82 & 55 & 9 & 8 & 10 & $79.7(72.6-85.0)$ & .391 \\
\hline RLL & 207 & 147 & 17 & 17 & 26 & $76.2(72.9-79.5)$ & .394 \\
\hline
\end{tabular}


TABLE 1. Continued

\begin{tabular}{|c|c|c|c|c|c|c|c|}
\hline & $\begin{array}{c}\text { No. } \\
\text { cases }\end{array}$ & $\begin{array}{c}\text { LODDS1 } \\
(-2.10<\text { LODDS } \\
\leq-1.01) \\
\end{array}$ & $\begin{array}{c}\text { LODDS2 } \\
(-1.01<\text { LODDS } \\
\leq-\mathbf{0 . 7 0}) \\
\end{array}$ & $\begin{array}{c}\text { LODDS3 } \\
(-0.70<\text { LODDS } \\
\leq-0.20) \\
\end{array}$ & $\begin{array}{c}\text { LODDS4 } \\
(-0.20<\text { LODDS } \\
\leq \mathbf{1 . 7 4}) \\
\end{array}$ & $\begin{array}{l}3-y \text { OS } \% \\
(95 \% \text { CI }) \\
\end{array}$ & $\begin{array}{c}P \text { value } \\
\text { (log-rank } \\
\text { test) } \\
\end{array}$ \\
\hline \multicolumn{8}{|l|}{ LNR } \\
\hline $0(\mathrm{LNR}=0)$ & 729 & 702 & 22 & 5 & 0 & $91.0(89.9-92.1)$ & $<.001$ \\
\hline $1(0<\mathrm{LNR} \leq 0.14)$ & 157 & 60 & 90 & 0 & 0 & $75.2(71.4-79.0)$ & \\
\hline $2(0.14<\mathrm{LNR} \leq 0.38)$ & 101 & 0 & 6 & 100 & 2 & $56.5(51.5-61.5)$ & \\
\hline $3(0.38<\mathrm{LNR} \leq 1)$ & 110 & 0 & 0 & 1 & 109 & $41.4(36.4-46.4)$ & \\
\hline \multicolumn{8}{|l|}{ LODDS } \\
\hline $1(-2.10<$ LODDS $\leq-1.01)$ & 762 & 762 & 0 & 0 & 0 & $89.9(88.7-91.1)$ & $<.001$ \\
\hline $2(-1.01<$ LODDS $\leq-0.70)$ & 118 & 0 & 118 & 0 & 0 & $78.4(74.4-82.4)$ & \\
\hline $3(-0.70<$ LODDS $\leq-0.20)$ & 106 & 0 & 0 & 106 & 0 & $56.0(50.9-61.1)$ & \\
\hline $4(-0.20<$ LODDS $\leq 1.74)$ & 111 & 0 & 0 & 0 & 111 & $41.0(36.1-45.9)$ & \\
\hline
\end{tabular}

LODDS, Log odds ratio; $O S$, overall survival; $C I$, confidence interval; TNM, Tumor, Node, Metastasis; seg, segmentectomy; Lob, lobectomy; $L U L$, left upper lobe; $L L L$, left lower lobe; $R U L$, right upper lobe; $R M L$, right middle lobe; $R L L$, right lower lobe; $L N R$, lymph node ratio.

Table 3. Among these 3 systems, the LODDS system showed greater LR, c-index, and lower AIC. According to our data, the LODDS system has the greatest ability to allow accurate prognostic stratification (by the linear trend test and by c-index) and homogeneity (by the LR test), along with greatest model fit (by the AIC). Based on the previous results, the next step is to see whether there is a good agreement between LNR and LODDS. As for the correlation between LNR and LODDS, the Spearman rank correlation coefficient was calculated to be 0.805 $(P<.001)$.

\section{DISCUSSION}

Despite the definition of the $\mathrm{N}$ stage, several studies have reported that LNR, the ratio between PLNs and total lymph nodes retrieved, is of prognostic value for lung cancer. ${ }^{3-5}$ In addition, LODDS, although not having been discussed in lung cancer, has been found of good prognostic value for gallbladder, ${ }^{10}$ head and neck, ${ }^{11}$ breast, ${ }^{12}$ colorectal, ${ }^{13,15,16}$ and gastric cancers. ${ }^{14}$ In clinical practice, the retrieved lymph nodes sometimes are not of sufficient quality for analyzing. Thus, compared with the number of metastatic lymph nodes, LNR and LODDS are thought to be more

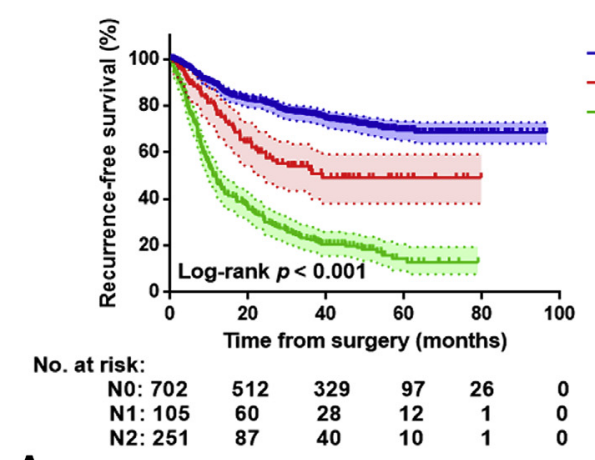

A
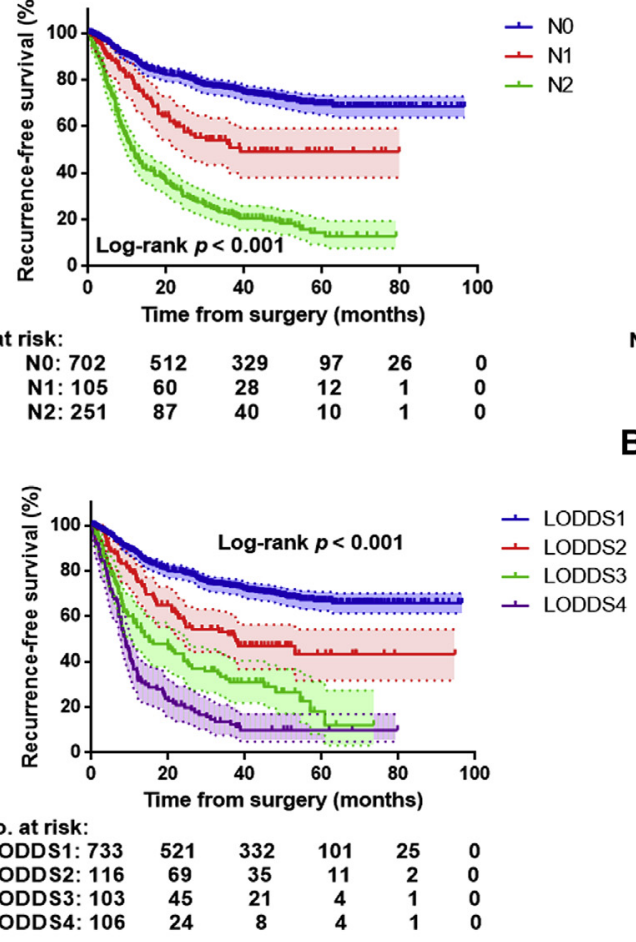

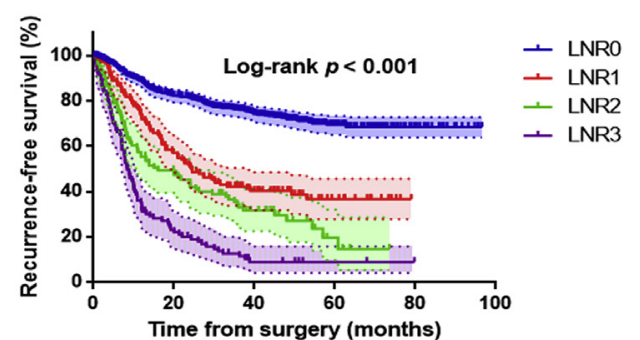

No. at risk:

$\begin{array}{lccccc}\text { LNRO: } 702 & 512 & 325 & 97 & 26 & 0\end{array}$

$\begin{array}{llcccc}\text { LNR1: } 146 & 77 & 40 & 15 & 1 & 0 \\ \text { LNR2: } 105 & 48 & 22 & 5 & 1 & 0 \\ \text { LNR3: } 105 & 23 & 7 & 3 & 1 & 0\end{array}$ 

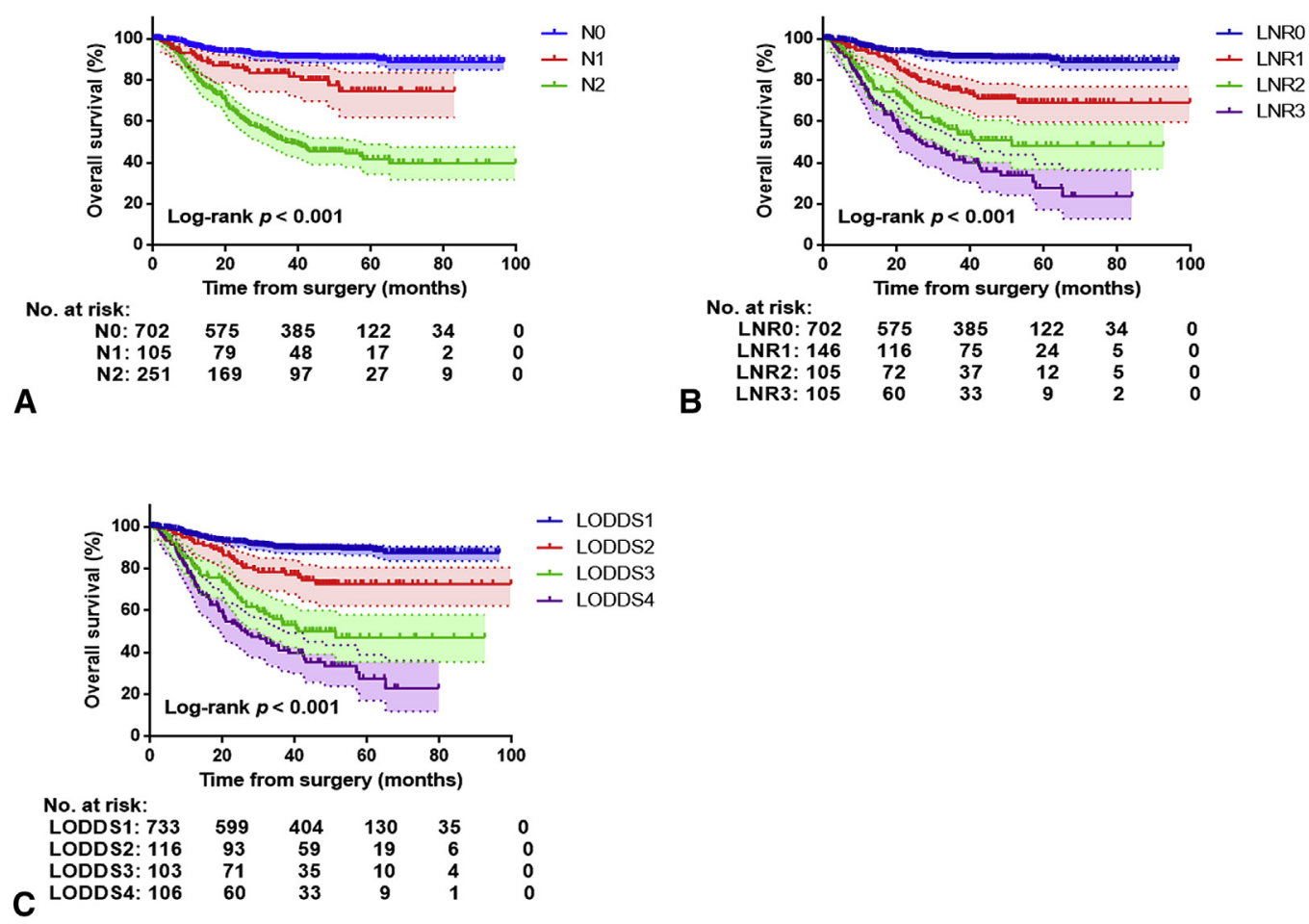

FIGURE 3. OS for $\mathrm{pN}$, LNR, and LODDS. A, OS for patients in different $\mathrm{pN}$ groups. B, OS for patients in different LNR groups. C, OS for patients in different LODDS groups. LNR, Lymph node ratio; LODDS, log odds ratio.

reliable. In this study, we included both LNR and LODDS and compared them with $\mathrm{pN}$ stage to find out whether LNR and LODDS are of prognostic value and furthermore, whether they have equal performance as the current $\mathrm{pN}$ staging system.

On the basis of our data, Table 1 shows that with the 3-year OS is associated significantly with $\mathrm{pN}$ stage $(P<.001)$, LNR $(P<.001)$, and LODDS $(P<.001)$. Patients in the lowest stage in each grouping systems had similar 3 -year OS $(91.0 \%$ for the N0 and LNR0 group and $89.9 \%$ for the LODDS1 group). However, the difference becomes increasingly greater when stages go greater $(50.8 \%$ for $\mathrm{N} 2$ group, $41.4 \%$ for LNR3 group, and $41.0 \%$ for LODDS4 group). This suggests the potential ability of the LNR and LODDS grouping system to distinguish patients' prognoses when they are in greater stages.

LODDS also can be used for considering the problem of false negativity. According to our results, the Spearman correlation test showed strong correlation between LNR and LODDS. Although LNR and LODDS appear to be different transformations of the same thing, LODDS can give us more information when LNR is equal. This raises an interesting question by Wang and colleagues ${ }^{15}$ : does patient A with 4 PLNs of 4 lymph nodes retrieved have the same prognosis as patient B with 20 PLNs of 20? Here, we may ask another question: does patient $\mathrm{C}$ with
0 PLNs of 4 have the same prognosis as patient $\mathrm{D}$ with 0 PLNs of 20? Intuitively, patient A should have a better prognosis than patient $\mathrm{B}$, and patient $\mathrm{C}$ should have a worse prognosis than patient D. Patient $\mathrm{A}$ and $\mathrm{B}$ have the same LNR, and so do patient C and D, but LODDS remains different (LODDS $=0.95$ for patient $\mathrm{A}, 1.61$ for patient $\mathrm{B},-0.95$ for patient $\mathrm{C}$, and -1.61 for patient $\mathrm{D}$, respectively). According to our grouping strategy, patient $\mathrm{A}$ and patient $\mathrm{B}$ should be in the LODDS4 group, patient $\mathrm{C}$ should be in the LODDS2 group, and patient $\mathrm{D}$ should be in the LODDS1 group. In this situation, patients with the same LNR (especially 0) can have different LODDSs; thus, they are in different groups and have different prognoses. Furthermore, LNR0 has the same meaning as N0, this means that N0 patients can have different LODDSs, thus different prognoses. It is of great value when making treatment strategies since the LODDS system has the ability to separate N0 patients into different groups to predict their prognoses because of the different number of lymph nodes retrieved. For example, when a patient has N0 disease but a high LODDS, a false-negative nodal status should be reasonably suspected, and a close follow-up is required. This also leads to another question that still hasn't reached a consensus: To resect how many lymph nodes would it be enough and how many would it be excess for surgical resectable non-small cell lung adenocarcinoma? Many 
TABLE 2. Univariable and multivariable Cox model of prognostic factors for overall survival

\begin{tabular}{|c|c|c|c|c|c|c|c|c|c|c|c|c|}
\hline & & $\begin{array}{c}\text { Univariable } \\
\text { analysis } \\
\end{array}$ & & & $\begin{array}{c}\text { Multivariable } \\
\text { analysis } 1 \\
\end{array}$ & & & $\begin{array}{c}\text { Multivariable } \\
\text { analysis } 2 \\
\end{array}$ & & & $\begin{array}{c}\text { Multivariable } \\
\text { analysis } 3\end{array}$ & \\
\hline & HR & $95 \% \mathrm{CI}$ & $P$ & HR & $95 \%$ CI & $P$ & HR & 95\% CI & $P$ & HR & 95\% CI & $P$ \\
\hline Sex & 1.477 & $1.129-1.932$ & .004 & 0.825 & $0.505-1.346$ & .441 & 0.795 & $0.490-1.290$ & .353 & 0.810 & $0.501-1.310$ & .391 \\
\hline Age & 1.007 & $0.994-1.021$ & .298 & & & & & & & & & \\
\hline Smoking status & 1.934 & $1.477-2.534$ & $<.001$ & 1.702 & $1.043-2.779$ & .033 & 1.755 & $1.082-2.847$ & .023 & 1.739 & $1.072-2.820$ & .025 \\
\hline Surgery type & & & $<.001$ & & & $<.001$ & & & $<.001$ & & & $<.001$ \\
\hline Wedge/seg & - & & & - & & & - & & & - & & \\
\hline Lob/bilob & 2.331 & $1.234-4.406$ & .009 & 2.697 & $1.290-5.636$ & .008 & 2.907 & $1.381-6.118$ & .005 & 3.035 & $1.442-6.386$ & .003 \\
\hline Pneumo & 10.398 & 4.489-24.08 & $<.001$ & 7.220 & $2.681-19.44$ & $<.001$ & 7.838 & $2.860-21.48$ & $<.001$ & 7.824 & $2.852-21.46$ & $<.001$ \\
\hline $\begin{array}{l}\text { Charlson comorbidity } \\
\text { index }\end{array}$ & & & .677 & & & & 0.359 & & .338 & & & .371 \\
\hline 0 & - & & & - & & & - & & & - & & \\
\hline 1 & 0.978 & $0.714-1.340$ & .978 & & & & & & & & & \\
\hline 2 & 0.691 & $0.324-1.474$ & .338 & & & & & & & & & \\
\hline 3 & 1.706 & $0.423-6.886$ & .453 & & & & & & & & & \\
\hline FEV $1 \%$ & 0.992 & $0.984-1.001$ & .096 & 0.997 & $0.987-1.006$ & .492 & 0.996 & $0.986-1.006$ & .382 & 0.996 & 0.986-1.006 & .397 \\
\hline $\begin{array}{l}\text { Adjuvant } \\
\text { chemotherapy }\end{array}$ & 3.187 & $2.394-4.243$ & $<.001$ & 0.633 & $0.419-0.955$ & .029 & 0.609 & $0.402-0.923$ & .019 & 0.585 & $0.388-0.883$ & .011 \\
\hline pT stage & & & $<.001$ & & & .003 & & & .003 & & & .001 \\
\hline 1 & - & & & - & & & - & & & - & & \\
\hline 2 & 2.557 & $1.886-3.467$ & $<.001$ & 1.289 & $0.902-1.843$ & .164 & 1.235 & $0.862-1.770$ & .251 & 1.274 & $0.892-1.821$ & .183 \\
\hline 3 & 6.732 & $4.383-10.34$ & $<.001$ & 2.605 & $1.473-4.608$ & .001 & 2.557 & $1.454-4.497$ & .001 & 2.806 & $1.629-4.833$ & $<.001$ \\
\hline 4 & 6.937 & $3.673-13.10$ & $<.001$ & 2.768 & $1.296-5.912$ & .009 & 2.620 & $1.226-5.600$ & .013 & 2.791 & $1.313-5.934$ & .008 \\
\hline pN stage & & & $<.001$ & & & .284 & & & & & & \\
\hline 0 & - & & & - & & & - & & & & & \\
\hline 1 & 2.454 & $1.479-4.071$ & .001 & 0.689 & $0.334-1.420$ & .312 & & & & & & \\
\hline 2 & 7.484 & $5.516-10.16$ & $<.001$ & 1.175 & $0.529-2.609$ & .691 & & & & & & \\
\hline pTNM stage & & & $<.001$ & & & $<.001$ & & & $<.001$ & & & $<.001$ \\
\hline 1 & - & & & - & & & - & & & - & & \\
\hline 2 & 4.621 & $2.875-7.427$ & $<.001$ & 4.377 & $2.165-8.850$ & $<.001$ & 4.185 & $2.085-8.403$ & $<.001$ & 3.396 & $1.790-6.440$ & $<.001$ \\
\hline 3 & 10.24 & $7.160-14.64$ & $<.001$ & 7.356 & $3.155-17.15$ & $<.001$ & 8.999 & $4.136-19.58$ & $<.001$ & 6.405 & $3.401-12.06$ & $<.001$ \\
\hline $\begin{array}{l}\text { Lymphovascular } \\
\text { invasion }\end{array}$ & 3.465 & $2.616-4.589$ & $<.001$ & 1.413 & $0.992-2.014$ & .056 & 1.345 & $0.946-1.914$ & .099 & 1.365 & $0.962-1.937$ & .081 \\
\hline Tumor location & & & .010 & & & .500 & & & .378 & & & .315 \\
\hline RUL & - & & & - & & & - & & & - & & \\
\hline RML & 0.668 & $0.319-1.399$ & .285 & 0.439 & $0.185-1.038$ & .061 & 0.439 & $0.185-1.039$ & .070 & 0.446 & $0.189-1.053$ & .066 \\
\hline RLL & 1.242 & $0.826-1.867$ & .298 & 0.931 & $0.593-1.464$ & .758 & 0.890 & $0.567-1.399$ & .614 & 0.909 & $0.579-1.429$ & .680 \\
\hline LUL & 1.227 & $0.852-1.765$ & .272 & 0.916 & $0.613-1.369$ & .669 & 0.866 & $0.577-1.299$ & .486 & 0.874 & $0.583-1.310$ & .514 \\
\hline LLL & 1.467 & $0.991-2.174$ & .056 & 0.959 & $0.599-1.535$ & .861 & 0.861 & $0.539-1.376$ & .531 & 0.872 & $0.546-1.392$ & .565 \\
\hline Number of LN resected & 1.705 & $1.272-2.285$ & $<.001$ & 1.054 & $0.742-1.499$ & .768 & 1.286 & $0.888-1.864$ & .184 & 1.326 & $0.909-1.934$ & .143 \\
\hline Number of positive LN & 1.117 & $1.101-1.134$ & $<.001$ & 1.031 & $1.002-1.061$ & .035 & 1.000 & $0.960-1.041$ & .994 & 0.992 & $0.952-1.034$ & .706 \\
\hline LNR & & & $<.001$ & & & & & & .073 & & & \\
\hline 0 & - & & & & & & - & & & & & \\
\hline 1 & 3.177 & $2.116-4.770$ & $<.001$ & & & & 0.733 & $0.367-1.462$ & .378 & & & \\
\hline 2 & 6.554 & $4.482-9.583$ & $<.001$ & & & & 1.118 & $0.536-2.334$ & .767 & & & \\
\hline 3 & 10.16 & $7.165-14.41$ & $<.001$ & & & & 1.703 & $0.720-4.027$ & .225 & & & \\
\hline LODDS & & & $<.001$ & & & & & & & & & .046 \\
\hline 1 & - & & & & & & & & & - & & \\
\hline 2 & 2.529 & $1.624-3.940$ & $<.001$ & & & & & & & 1.165 & $0.668-2.032$ & .590 \\
\hline 3 & 5.872 & $4.060-8.494$ & $<.001$ & & & & & & & 1.682 & $0.944-2.996$ & .078 \\
\hline 4 & 9.081 & $6.504-12.68$ & $<.001$ & & & & & & & 2.658 & $1.296-5.449$ & .008 \\
\hline
\end{tabular}

LNR was categorized into 4 groups: LNR0 (LNR $=0)$, LNR1 $(0<$ LNR $\leq 0.14)$, LNR2 $(0.14<$ LNR $\leq 0.38)$ and LNR3 $(0.38<$ LNR $\leq 1)$. LODDS was categorized into 4 groups: LODDS1 $(-2.10<$ LODDS $\leq-1.01)$, LODDS2 $(-1.01<$ LODDS $\leq-0.70)$, LODDS3 $(-0.70<$ LODDS $\leq-0.20)$, and LODDS4 $(-0.20<$ LODDS $\leq 1.74)$. $H R$, Hazard ratio; CI, confidence interval; seg, segmentectomy; Lob, lobectomy; Bilob, bilobectomy; Pneumo, pneumonectomy; FEVI\%, percent of forced expiratory volume in 1 second predicted; $p T$, pathologic $\mathrm{T}$ stage; $p N$, pathologic $\mathrm{N}$ stage; $p T N M$, pathologic TNM stage; $R U L$, right upper lobe; $R M L$, right middle lobe; $R L L$, right lower lobe; $L U L$, left upper lobe; $L L L$, left lower lobe; $L N$, lymph node; $L N R$, lymph node ratio; $L O D D S$, log odds ratio. 
TABLE 3. Measurement of homogeneity, discriminatory ability, and monotonicity of pN, LNR, and LODDS systems

\begin{tabular}{lcccc}
\hline & $\begin{array}{c}\text { Linear trend } \\
\text { test }\left(\chi^{\mathbf{2}}\right)\end{array}$ & $\begin{array}{c}\text { Likelihood } \\
\text { ratio test }\left(\boldsymbol{\chi}^{\mathbf{2}}\right)\end{array}$ & HC index & AIC \\
\hline $\mathrm{pN}$ & 222.5 & 255 & 0.8016 & 619.1 \\
$\mathrm{LNR}$ & 255.8 & 257 & 0.8019 & 619.2 \\
LODDS & 249.9 & 259 & 0.8025 & 617.1 \\
\hline
\end{tabular}

$p N$, Pathologic $\mathrm{N}$ stage; $L N R$, lymph node ratio; LODDS, log odds ratio; $H C$ index, Harrell C index; AIC, Akaike information criterion.

studies have contributed to this topic and it still needs further proving. ${ }^{17-20}$

In conclusion, LNR and LODDS are of good prognostic value in predicting patients' prognoses. They can be taken into consideration for clinicians when predicting patients' prognosis and making personalized therapeutic strategies. Particularly, LODDS can be taken into consideration the problem of false-negativity when none of the resected lymph nodes is positive. And of course, we can use all the 3 systems together to have more information. With few studies discussing the value of LODDS in lung cancer, we hope our study contributes to the literature and more data come out in the future to gain a better understanding of this topic.

\section{Conflicts of Interest Statement}

Authors have nothing to disclose with regard to commercial support.

\section{References}

1. Goldstraw P, Crowley J, Chansky K, Giroux DJ, Groome PA, Rami-Porta R, et al. The IASLC Lung Cancer Staging Project: proposals for the revision of the TNM stage groupings in the forthcoming (seventh) edition of the TNM Classification of malignant tumours. J Thorac Oncol. 2007;2:706-14.

2. Goldstraw P, Chansky K, Crowley J, Rami-Porta R, Asamura H, Eberhardt WE, et al. The IASLC Lung Cancer Staging Project: Proposals for Revision of the TNM Stage Groupings in the Forthcoming (Eighth) Edition of the TNM Classification for Lung Cancer. J Thorac Oncol. 2016;11:39-51.

3. Sun G, Xue L, Wang M, Zhao X. Lymph node ratio is a prognostic factor for non-small cell lung cancer. Oncotarget. 2015;6:33912-8.

4. Wu CF, Wu CY, Fu JY, Wang CW, Liu YH, Hsieh MJ, et al. Prognostic value of metastatic N1 lymph node ratio and angiolymphatic invasion in patients with pathologic stage IIA non-small cell lung cancer. Medicine (Baltimore). 2014;93:e102.

5. Taylor MD, Lapar DJ, Thomas CJ, Persinger M, Stelow EB, Kozower BD, et al. Lymph node ratio predicts recurrence and survival after R0 resection for non-small cell lung cancer. Ann Thorac Surg. 2013;96:1163-70.
6. Urban D, Bar J, Solomon B, Ball D. Lymph node ratio may predict the benefit of postoperative radiotherapy in non-small-cell lung cancer. J Thorac Oncol. 2013;8:940-6

7. Nwogu CE, Groman A, Fahey D, Yendamuri S, Dexter E, Demmy TL, et al Number of lymph nodes and metastatic lymph node ratio are associated with survival in lung cancer. Ann Thorac Surg. 2012;93:1614-9.

8. Jonnalagadda S, Arcinega J, Smith C, Wisnivesky JP. Validation of the lymph node ratio as a prognostic factor in patients with $\mathrm{N} 1$ nonsmall cell lung cancer. Cancer. 2011;117:4724-31

9. Wisnivesky JP, Arciniega J, Mhango G, Mandeli J, Halm EA. Lymph node ratio as a prognostic factor in elderly patients with pathological N1 non-small cell lung cancer. Thorax. 2011;66:287-93.

10. Amini N, Kim Y, Wilson A, Margonis GA, Ethun CG, Poultsides G. Prognostic implications of lymph node status for patients with gallbladder cancer: a multiinstitutional study. Ann Surg Oncol. 2016;23:3016-23.

11. Yildiz MM, Petersen I, Eigendorff E, Schlattmann P, Guntinas-Lichius O Which is the most suitable lymph node predictor for overall survival after primary surgery of head and neck cancer: $\mathrm{pN}$, the number or the ratio of positive lymph nodes, or log odds? J Cancer Res Clin Oncol. 2016;142: 885-93.

12. Wen J, Ye F, He X, Li S, Huang X, Xiao X, et al. Development and validation of a prognostic nomogram based on the log odds of positive lymph nodes (LODDS) for breast cancer. Oncotarget. 2016;7:21046-53.

13. Lee CW, Wilkinson KH, Sheka AC, Leverson GE, Kennedy GD. The log odds of positive lymph nodes stratifies and predicts survival of high-risk individuals among stage iii rectal cancer patients. Oncologist. 2016;21: 425-32.

14. Jian-Hui C, Shi-Rong C, Hui W, Si-le C, Jian-Bo X, Er-Tao Z, et al. Prognostic value of three different lymph node staging systems in the survival of patients with gastric cancer following D2 lymphadenectomy. Tumour Biol. 2016;37: 11105-13.

15. Wang J, Hassett JM, Dayton MT, Kulaylat MN. The prognostic superiority of log odds of positive lymph nodes in stage III colon cancer. J Gastrointest Surg. 2008; 12:1790-6.

16. Song YX, Gao P, Wang ZN, Tong LL, Xu YY, Sun Z, et al. Which is the mos suitable classification for colorectal cancer, log odds, the number or the ratio of positive lymph nodes? PLoS One. 2011;6:e28937.

17. Ludwig MS, Goodman M, Miller DL, Johnstone PA. Postoperative survival and the number of lymph nodes sampled during resection of node-negative non-small cell lung cancer. Chest. 2005;128:1545-50.

18. Varlotto JM, Recht A, Nikolov M, Flickinger JC, Decamp MM. Extent of lymphadenectomy and outcome for patients with stage I nonsmall cell lung cancer. Cancer. 2009;115:851-8.

19. Saji H, Tsuboi M, Yoshida K, Kato Y, Nomura M, Matsubayashi J, et al. Prognostic impact of number of resected and involved lymph nodes at complete resection on survival in non-small cell lung cancer. J Thorac Oncol. 2011;6: 1865-71.

20. Yang M, Cao H, Guo X, Zhang T, Hu P, Du J, et al. The number of resected lymph nodes (nLNs) combined with tumor size as a prognostic factor in patients with pathologic N0 and Nx non-small cell lung cancer. PLoS One. 2013;8: e73220.

Key Words: prognostic value, lymph node ratio, log odds ratio, lung adenocarcinoma 
TABLE E1. Distribution between pN and LNR

\begin{tabular}{lcccc}
\hline & LNR0 & LNR1 & LNR2 & LNR3 \\
\hline N0 & 729 & 0 & 0 & 0 \\
N1 & 0 & 77 & 20 & 11 \\
N2 & 0 & 73 & 88 & 99 \\
\hline$p N$, Pathologic N stage; $L N R$, lymph node ratio. & &
\end{tabular}

TABLE E2. A comparison between single or multiple lymph node stations involved in patients with $\mathrm{N} 1$ and $\mathrm{N} 2$ diseases

\begin{tabular}{lccr}
\hline & $\begin{array}{c}\text { Single } \\
\text { station involved }\end{array}$ & $\begin{array}{c}\text { Multiple } \\
\text { stations involved }\end{array}$ & $\boldsymbol{P}$ value \\
\hline $\mathrm{N} 1(\mathrm{n}=63)$ & $75(69.4 \%)$ & $33(30.6 \%)$ & $<.001$ \\
$\mathrm{~N} 2(\mathrm{n}=165)$ & $53(20.4 \%)$ & $207(79.6 \%)$ & \\
\hline$P$ value was calculated with Pearson $\chi^{2}$ test. & &
\end{tabular}

